\title{
ON THE VALENCE OF THE SUM OF TWO CONVEX FUNCTIONS
}

\author{
D. STYER ${ }^{1}$ AND D. WRIGHT
}

\begin{abstract}
In this paper it is shown that the sum of two starlike functions may have an infinite number of zeros and that the sum of two convex functions may be at least three-valent. Furthermore, the convex sum of two odd convex functions is studied.
\end{abstract}

1. Introduction. Let $S$ denote the class of functions $f(z)=z+\sum_{2}^{\infty} a_{n} z^{n}$ which are analytic and univalent in the unit disc $U=\{z:|z|<1\}$. Let $S^{*}$, $K$, and $C$ denote the subclasses of $S$ whose members are starlike, convex, and close-to-convex respectively in $U$. There have been a number of questions raised regarding the sum of two members of a given subclass of $S$ [1]. In particular, it had been conjectured [3, Problem 6.11], [8, Problem 21] that $f+g \in S^{*}$ for $f$ and $g$ in $K$. MacGregor [4] has shown this to be false by producing a pair of functions in $K$ whose sum is two-valent, and Goodman [1, p. 1045] has suggested that the sum of two convex functions is at most two-valent.

In the present note we show by geometric considerations that Goodman's conjecture is also false, and we indicate that $f+g$ may very likely be infinite-valent for some $f, g \in K$. We also exhibit two starlike functions whose sum is infinite-valent, answering in the negative a question raised in [2]. However, if $f$ and $g$ are odd convex functions, then $\lambda f+(1-\lambda) g \in C$, $0<\lambda<1$, and we show that this function need not be starlike for $\lambda \neq 1$ discussion of the case $\lambda=\frac{1}{2}$ raises a question concerning odd convex functions.

Since $f \in K$ iff $z f^{\prime} \in S^{*}[5$, p. 223], we can study convex functions in terms of starlike functions, in which the geometric complexities are more apparent. If $f \in S^{*}$ and the image of $(0,1)$ under $f$ crosses the imaginary axis, then $g(z)=\overline{f(\bar{z})} \in S^{*}$ and $f+g$ has a zero in $(0,1)$. If $F$ and $G$ are the corresponding convex functions, then $F+G$ has a critical point in $(0,1)$, and thus is not univalent. This approach is used in each of the following examples.

Received by the editors December 16, 1971.

AMS (MOS) subject classifications (1970). Primary 30A32.

Key words and phrases. Convex, starlike, odd univalent functions, convex sums.

${ }^{1}$ Supported in part by a Charles Phelps Taft Postdoctoral Fellowship at the University of Cincinnati.

ç American Mathematical Society 1973 
2. Example 1. There exist functions $f, g \in S^{*}$ such that $f+g$ has infinitely many zeros in $(0,1)$.

We shall need the well-known fact [6] that $f \in S^{*}$ if and only if

$$
f(z)=z \exp \left\{-2 \int_{-\pi}^{\pi} \log \left(1+z e^{-i t}\right) d \mu(t)\right\},
$$

where $\mu$ is an increasing function on $[-\pi, \pi]$ with $\mu(-\pi)=0, \mu(\pi)=1$. We shall construct inductively a sequence of functions $f_{n} \in S^{*}$ with the property that the image of $(0,1)$ under $f_{n}$ intersects the imaginary axis at least $n$ times. Since $S^{*}$ is a compact normal family we may assume without loss of generality that $f_{n} \rightarrow f \in S^{*}$ uniformly on compact subsets of $U$. Letting $g_{n}(z)=\overline{f_{n}(\bar{z})} \in S^{*}, g_{n} \rightarrow g \in S^{*}$, we see that $f_{n}+g_{n}$ has at least $n$ zeros in $(0,1)$. The construction will be such that $f+g$ will have infinitely many zeros in $(0,1)$.

From (1), $\operatorname{Re}\{f(r) / r\}=0$ for some $r \in(0,1)$ if

$$
\operatorname{Im}\left\{\int_{-\pi}^{\pi} \log \left(1+r e^{-i t}\right) d \mu(t)\right\}=-\frac{\pi}{4} .
$$

Thus, we shall find a sequence of functions $\mu_{n}$, increasing on $[-\pi, \pi]$ with $\mu(-\pi)=0, \mu(\pi)=1$, such that

$$
\Lambda_{n}(r)=\frac{\pi}{4}+\int_{-\pi}^{\pi} \arg \left(1+r e^{-i t}\right) d \mu_{n}(t)
$$

has at least $n$ zeros in $(0,1)$. We shall select sequences $\left\{\theta_{k}\right\}$ and $\left\{\alpha_{k}\right\}, \alpha_{k}>0$, $k=1,2, \cdots$, such that for each $n>0, \pi / 2<\theta_{1}<\theta_{2}<\cdots<\theta_{n}<\pi$, and $\sum_{1}^{n} \alpha_{k}<1$. We thus determine step functions $\mu_{n}, \mu_{n}(-\pi)=0$, with jump $\alpha_{k}$ at $(-1)^{k+1} \theta_{k}$ and jump $1-\sum_{1}^{n} \alpha_{k}$ at $\pi$. For these functions (2) becomes

Furthermore,

$$
\Lambda_{n}(r)=\frac{\pi}{4}+\sum_{k=1}^{n} \alpha_{k} \arg \left(1+r \exp \left((-1)^{k} i \theta_{k}\right)\right)
$$

$$
\Lambda_{n}(1)=\lim _{r \rightarrow 1^{-}} \Lambda_{n}(r)=\left(\frac{1}{2}\right)\left\{\frac{\pi}{2}+\sum_{k=1}^{n}(-1)^{k} \alpha_{k} \theta_{k}\right\} .
$$

The following relation between $\Lambda_{n}(1)$ and $\Lambda_{n+1}(1)$ will be useful:

$$
(-1)^{n+1} 2 \Lambda_{n+1}(1)=(-1)^{n+1} 2 \Lambda_{n}(1)+\alpha_{n+1} \theta_{n+1} \text {. }
$$

Choose $\theta_{1}>\pi / 2$, and pick $\alpha_{1}$ such that $\pi / 2 \theta_{1}<\alpha_{1}<3 \pi / 2\left(\pi+\theta_{1}\right)$, or equivalently, such that $\pi\left(1-\alpha_{1}\right)>\alpha_{1} \theta_{1}-\pi / 2>0$. Then $\alpha_{1} \arg \left(1+\exp \left(-i \theta_{1}\right)\right)=$ $-\theta_{1} \alpha_{1} / 2<-\pi / 4$, so we may choose $r_{1}$ sufficiently near 1 that

$$
\Lambda_{1}\left(r_{1}\right)=\pi / 4+\alpha_{1} \arg \left(1+r_{1} \exp \left(-i \theta_{1}\right)\right)<0 .
$$

Thus, $\Lambda_{1}(0)=\pi / 4, \Lambda_{1}\left(r_{1}\right)<0$, so $\Lambda_{1}$ has at least one zero in $\left(0, r_{1}\right)$. 
Now suppose that for $k=1,2, \cdots, n$, numbers $\alpha_{k}, \theta_{k}$, and $r_{k}$ have been selected satisfying the conditions

$$
\begin{gathered}
\alpha_{k}>0, \quad \sum_{1}^{n} \alpha_{k}<1, \\
\pi / 2<\theta_{1}<\theta_{2}<\cdots<\theta_{n}<\pi, \quad 0<r_{1}<r_{2}<\cdots<r_{n}<1, \\
\pi\left(1-\sum_{1}^{n} \alpha_{k}\right)>(-1)^{n} 2 \Lambda_{n}(1)>0, \\
(-1)^{l} \Lambda_{n}\left(r_{l}\right)>0, \quad l=1,2, \cdots, n .
\end{gathered}
$$

From (5), we may choose $\theta_{n+1} \in\left(\theta_{n}, \pi\right)$ sufficiently near $\pi$ that

$$
\frac{(-1)^{n} 2 \Lambda_{n}(1)}{\theta_{n+1}}<\frac{(-1)^{n} 2 \Lambda_{n}(1)+\pi\left(1-\sum_{1}^{n} \alpha_{k}\right)}{\pi+\theta_{n+1}}<1-\sum_{1}^{n} \alpha_{k},
$$

and from (6) we may also insist that

$$
\begin{array}{r}
(-1)^{l}\left\{\Lambda_{n}\left(r_{l}\right)+\arg \left(1+r_{l} \exp \left((-1)^{n+1} i \theta_{n+1}\right)\right)\right\}=(-1)^{l} \Lambda_{n+1}\left(r_{l}\right)>0, \\
l=1,2, \cdots, n,
\end{array}
$$

since $\arg \left(1+r_{l} \exp \left((-1)^{n+1} i \theta\right)\right) \rightarrow 0$ as $\theta \rightarrow \pi^{-}$. Select $\alpha_{n+1}$ in the open interval determined by the left-hand inequality in (7), or equivalently, such that

$$
\pi\left(1-\sum_{1}^{n+1} \alpha_{k}\right)>(-1)^{n+1} 2 \Lambda_{n+1}(1)>0 .
$$

It is perhaps simplest to use (4) to obtain (9) from (7).

By (3) and the right-hand inequality in (9), it is immediate that for some $r_{n+1} \in\left(r_{n}, 1\right)$ we have $(-1)^{n+1} 2 \Lambda_{n+1}\left(r_{n+1}\right)>0$. This, together with (8), guarantees that $(-1)^{l} \Lambda_{n+1}\left(r_{l}\right)>0$ for $l=1,2, \cdots, n+1$, and thus that $\Lambda_{n+1}(r)$ has at least $l$ zeros in $\left(0, r_{l}\right), l=1,2, \cdots, n+1$.

The functions $f_{n}$ are so determined that $f_{m}+g_{m}$ has at least $n$ zeros in $\left(0, r_{n}\right)$ for $n \leqq m$, and thus $f+g$ has infinitely many zeros in $(0,1)$, by Hurwitz's theorem.

3. Example 2. There exist functions $F, G \in K$ such that $F+G$ is at least three-valent in $U$.

Let $f, g \in S^{*}$ be the functions determined in Example 1, and let $F$ and $G$ be the corresponding members of $K$ defined by $f=z F^{\prime}, g=z G^{\prime}$. For $r \in$ $(0,1)$,

$$
F(r)+G(r)=\int_{0}^{r} \frac{f(\rho)+g(\rho)}{\rho} d \rho .
$$


Suppose the positive real zeros of $f+g$ are $\rho_{k}, k=1,2, \cdots, \rho_{1}<\rho_{2}<\cdots$. Since $z F^{\prime}(z)+z G^{\prime}(z)=f(z)+g(z), F^{\prime}+G^{\prime}$ has a zero at each point $\rho_{k}$, $k=1,2, \cdots$. If $\rho_{k}$ is a zero of order $n_{k}>1$, then $F+G$ is $\left(n_{k}+1\right)$-valent in each neighborhood of $\rho_{k}$. Thus $F+G$ is at least 3-valent. If $F^{\prime}+G^{\prime}$ has a zero of order 1 at each point $\rho_{k}$, then $F(r)+G(r)$ increases in $\left(0, \rho_{1}\right)$, decreases in $\left(\rho_{1}, \rho_{2}\right)$, and increases again in $\left(\rho_{2}, \rho_{3}\right)$. Furthermore, $F\left(\rho_{2}\right)+$ $G\left(\rho_{2}\right)>0$, since $\operatorname{Re}\{h(z) / z\}>\frac{1}{2}$ for all $h \in K$ [7]. It follows that for $\varepsilon>0$ sufficiently small each number in the interval $\left(F\left(\rho_{2}\right)+G\left(\rho_{2}\right), F\left(\rho_{2}\right)+\right.$ $\left.G\left(\rho_{2}\right)+\varepsilon\right)$ has at least three distinct pre-images in $\left(0, \rho_{3}\right)$, so that $F+G$ is at least three-valent.

We note that $F+G$ has a critical point at each $\rho_{k}, k=1,2, \cdots$. It seems likely that the function $f$ could be constructed in such a way that the curve $F(r)+G(r), r \in[0,1)$, crosses some point on the real axis infinitely often, and so we expect that there may well be two members of $K$ whose sum has valence infinity.

4. Let $K^{(2)}$ denote the class of odd convex functions and let $H_{\lambda}=$ $\left\{\lambda f+(1-\lambda) g: f, g \in K^{(2)}\right\}, 0<\lambda<1$.

TheOREM 1. For all $\lambda \in(0,1), H_{\lambda} \subset C$.

Proof. If $f \in K^{(2)}$, then $h(z)=z f^{\prime}(z)$ is odd and starlike. By a result of Strohhacker [7], $\operatorname{Re}\left\{f^{\prime}(z)\right\}=\operatorname{Re}\{h(z) / z\}>1$. Thus, $\operatorname{Re}\left\{\lambda f^{\prime}+(1-\lambda) g^{\prime}\right\}>\frac{1}{2}$ for all $f, g \in K^{(2)}$, so each member of $H_{j}$ is close-to-convex with respect to the identity function.

THEOREM 2. If $\lambda \neq \frac{1}{2}$, then $H_{\lambda} \phi S^{*} . H_{1 / 2} \subset S^{*}$ if and only if $|\operatorname{Im}\{f(z) \mid z\}|<$ $\pi / 4$ for all $f \in K^{(2)}$, and all $z \in U$.

REMARK. We were unable to show that $|\operatorname{Im}\{f(z) / z\}|<\pi / 4$ for all $f \in K^{(2)}$ and all $z \in U$. We suspect that this is true. In fact we believe that $f(z) / z$ is subordinate to $(1 / 2 z) \ln [(1+z) /(1-z)]$.

Proof. We shall first prove the second statement of Theorem 2 . It is known [7] that if $f \in K^{(2)}$, then $\operatorname{Re}\{f(z) / z\}>\pi / 4, z \in U$, and that the largest disc centered at the origin and contained in $f(U)$ has radius $\rho \geqq \pi / 4$. A simple subordination argument shows that $\rho=\pi / 4$ only for rotations of $f(z)=\left(\frac{1}{2}\right) \ln [(1+z) /(1-z)]$.

Suppose $|\operatorname{Im}\{f(z) / z\}|<\pi / 4$ for all $f \in K^{(2)}, z \in U$. Since $K^{(2)}$ is a compact normal family, we may assume, without loss of generality, that the functions under consideration are analytic on the boundary of $U$. Furthermore, since $K^{(2)}$ is rotationally invariant it suffices to show that if $|\operatorname{Im}\{f(1)\}|<\pi / 4$ for all $f \in K^{(2)}$ which are analytic on $\partial U$, then

$$
\operatorname{Re}\left\{\left[f^{\prime}(1)+g^{\prime}(1)\right] /[f(1)+g(1)]\right\}>0
$$


for all $f, g \in K^{(2)}$ which are analytic on $\partial U$. From the above restrictions it follows that

$$
\arg [f(1)+g(1)] \geqq \arg [f(1)-\pi i / 4]
$$

(see Figure 1). Let $\Gamma$ be the circle with center $f(1)$ and radius $\pi / 4$. If $T$ is the point where the lower tangent from 0 to $\Gamma$ meets $\Gamma$, then

$$
\arg T \leqq \arg [f(1)-\pi i / 4]
$$

The vector from $f(1)$ to $f(1)+i f^{\prime}(1)$ is the vector tangent to the curve $f\left(e^{i \theta}\right)$ at $\theta=0$. Since $f$ is convex and bounded, by paragraph 1 of this proof, we see that the argument of this vector must be less than arg $T+\pi$. That is, $\arg i f^{\prime}(1)<\arg T+\pi$, or equivalently,

$$
\arg f^{\prime}(1)<\arg T+\pi / 2 .
$$

From (10), (11), (12) it follows that

$$
\arg \left\{f^{\prime}(1) /(f(1)+g(1))\right\}<\pi / 2,
$$

and a similar argument yields

$$
\arg \left\{f^{\prime}(1) /(f(1)+g(1))\right\}>-\pi / 2 .
$$

Thus, $\operatorname{Re}\left\{f^{\prime}(1) /(f(1)+g(1))\right\}>0$, and by interchanging $f$ and $g$, $\operatorname{Re}\left\{g^{\prime}(1) /(f+g)(1)\right\}>0$, so $\operatorname{Re}\left\{\left(f^{\prime}(1)+g^{\prime}(1)\right) /(f(1)+g(1))\right\}>0$.

Conversely, suppose $f \in K^{(2)}, f$ analytic on $\partial U$, and $\operatorname{Im}\{f(1)\}-\pi / 4>0$.

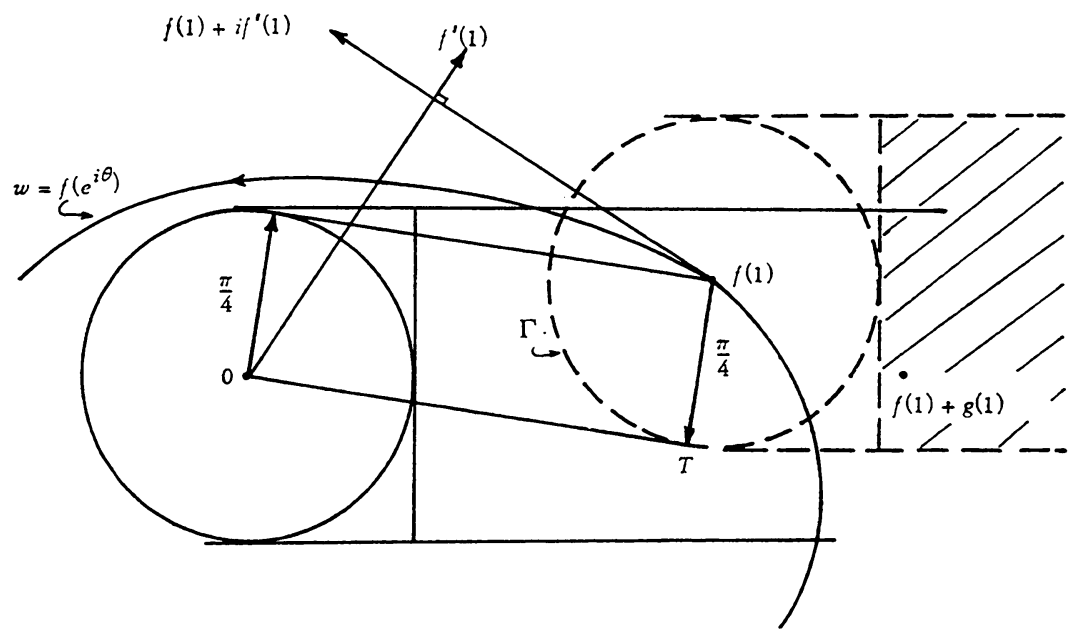

FIGURE 1 
For $g(z)=\left(\frac{1}{2}\right) \ln [(1+z) /(1-z)]$, we obtain

and

$$
\lim _{\theta \rightarrow 0^{-}} \operatorname{Re}\left\{f\left(e^{i \theta}\right)+g\left(e^{i \theta}\right)\right\}=+\infty,
$$

$$
\lim _{\theta \rightarrow 0^{-}} \operatorname{Im}\left\{f\left(e^{i \theta}\right)+g\left(e^{i \theta}\right)\right\}=\operatorname{Im}\{f(1)\}-\pi / 4>0 .
$$

It is geometrically apparent that $(f+g) / 2$ is not starlike, and straightforward calculations show that $(\partial / \partial \theta) \arg \left[\left(f\left(e^{i \theta}\right)+g\left(e^{i \theta}\right)\right]<0\right.$ for $\theta<0, \theta$ sufficiently small.

By a similar argument we shall now prove the first part of Theorem 2 . Suppose $\lambda>\frac{1}{2}$ and let

Now,

$$
f_{\theta}(z)=\frac{1}{2} e^{-i \theta} \ln \left(\left(1+z e^{i \theta}\right) /\left(1-z e^{i \theta}\right)\right) .
$$

$$
\operatorname{Im}\{f(1)\}=-\frac{\sin \theta}{2} \ln \left|\frac{1+e^{i \theta}}{1-e^{i \theta}}\right|+\frac{\pi}{4}(\cos \theta) \arg \left(\frac{1+e^{i \theta}}{1-e^{i \theta}}\right),
$$

and a computation shows that

$$
\lim _{\theta \rightarrow 0^{+}} \operatorname{Im}\left\{f_{\theta}(1)\right\}=\frac{\pi}{4} .
$$

Choose $\theta>0$ sufficiently near zero that $\operatorname{Im}\left\{2 \lambda f_{\theta}(1)\right\}>\pi / 4$. If we let $f=f_{\theta}$ and $g(z)=\left(\frac{1}{2}\right) \ln [(1+z) /(1-z)]$, we see, as in the previous part of the proof, that $\lambda f+(1-\lambda) g \notin S^{*}$.

\section{REFERENCES}

1. A. W. Goodman, Open problems on univalent and multivalent functions, Bull. Amer. Math. Soc. 74 (1968), 1035-1050. MR 38 \#315.

2. - The valence of sums and products, Canad. J. Math. 20 (1968), 1173-1177.

3. W. K. Hayman, Research problems in function theory, Athlone Press [University of London], London, 1967. MR 36 \#359.

4. T. H. MacGregor, The univalence of a linear combination of convex mappings, $\mathrm{J}$. London Math. Soc. 44 (1969), 210-212. MR 38 \#4665.

5. Z. Nehari, Conformal mapping, McGraw-Hill, New York, 1952. MR 13, 640.

6. M. S. Robertson, On the theory of univalent functions, Ann. of Math. (2) 37 (1936), 374-408.

7. E. Strohhacker, Beitrage zur Theorie der schlichten Funktionen, Math. Z. 37 (1933), 356-380.

8. Problem 21, Classical function theory problems, Bull. Amer. Math. Soc. 68 (1962), 21-24.

Department of Mathematics, University of Cincinnati, Cincinnati, Ohio 45221 